\title{
Route of Administration
}

National Cancer Institute

\section{Source}

National Cancer Institute. Route of Administration. NCI Thesaurus. Code C38114.

Designation of the part of the body through which or into which, or the way in which, the medicinal product is intended to be introduced. In some cases a medicinal product can be intended for more than one route and/or method of administration. 